\title{
Topological Entropy of Free Semigroup Actions Generated by Proper Maps for Noncompact Subsets
}

\author{
Xiaoyi Xie and Dongkui Ma*
}

\begin{abstract}
In this paper, we introduce three notions of topological entropy of a free semigroup action generated by proper maps for noncompact subsets, which extends the notions defined by $\mathrm{Ju}$ et al. [13 and $\mathrm{Ma}$ et al. 17]. By using the one-point compactification as a bridge, we study the relations of the entropies between two dynamical systems. We then introduce three skew-product transformations, and for a particular subset, the relationship between the upper capacity topological entropy of a free semigroup action generated by proper maps, and the upper capacity topological entropy of a skew-product transformation is given. As applications, we examine the multifractal spectrum of a locally compact separable metric space, and it is shown that the irregular set has full upper capacity topological entropy of a free semigroup action generated by proper maps.
\end{abstract}

\section{Introduction}

Topological entropy, which plays a significant role in topological dynamical systems, was first introduced by Adler, Konheim and McAndrew [1]. Using spanning sets and separated sets, Bowen [5] then defined topological entropy for a uniformly continuous map on a metric space, which extended the definition of Adler, Konheim and McAndrew. After that, Bowen [6] introduced the topological entropy on noncompact sets by using the approach of the definition of the Hausdorff dimension.

The classical Carathéodory construction in the general measure theory was originated by Carathéodory in [8. Pesin [21] proposed a new structure extending the classical Carathéodory construction, which we call Carathéodory-Pesin structure or C-P structure for short. The $\mathrm{C}-\mathrm{P}$ structure is a powerful tool to study dimension theory and dynamical systems, which produces various characteristics of dimension type. For example, Hausdorff dimension, topological entropy, etc. Further, it is very helpful to study the topological entropy of noncompact subsets. Topological entropy for noncompact sets can

Received May 15, 2021; Accepted September 12, 2021.

Communicated by Jenn-Nan Wang.

2020 Mathematics Subject Classification. 37A35, 37B40.

Key words and phrases. topological entropy, proper map, free semigroup actions, skew-product transformations, irregular set, multifractal spectrum.

*Corresponding author. 
be used to investigate multifractal spectra, saturated sets and irregular sets of dynamical systems, see, for example, $2,6,10,21,23$.

In recent years, people have paid more attention to the study of free semigroup actions. On the one hand, it is needed by some other disciplines, such as in physics, sometimes it is necessary to allow the system that describes the real events to readjust with time to match the inevitable experimental errors [14]. On the other hand, some dynamic system theories are closely related to it, for example in the case of a foliation on a manifold and a pseudo-group of holonomy maps, such as the one studied by Ghys, Langevin and Walczak in [12. In [12], they introduced a notion of topological entropy for finitely generated pseudo-groups of continuous maps. Biś [3] and Bufetov [7] introduced the notion of the topological entropy for free semigroup actions on a compact metric space, respectively. Related studies include $9,16,22,25,26$, etc.

By using the C-P structure, Ma et al. [19] and Ju et al. [13 introduced topological entropy of a free semigroup action in different ways, Xiao et al. gave two new notions of topological pressure of a free semigroup action in 27] and 28] respectively, Biś et al. [4] studied the topological entropy and upper Carathéodory capacity of a semigroup action.

For a topological space, Patrão 20] introduced the topological entropy of proper maps, on this basis, Ma et al. 17] introduced three notions of topological entropy of proper maps by using the C-P structure. Moreover, Tian et al. 24], Ma and Fan [18, Li et al. [15] and so on further studied the topological entropy and topological pressure of proper maps.

Inspired by the above notions, the main purpose of this paper is to introduce the topological entropy, lower capacity topological entropy (LCTE) and upper capacity topological entropy (UCTE) of a free semigroup action generated by $m$ proper maps for noncompact subsets by using the $\mathrm{C}-\mathrm{P}$ structure. Moreover, some further properties of the topological entropy, LCTE and UCTE for a locally compact separable metric space (LCSMS) are studied. We also give some applications of these entropies.

This paper is organized as follows. In Section 2, we give some preliminaries. In Section 3 , by using the $\mathrm{C}-\mathrm{P}$ structure we introduce the notions of the topological entropy, LCTE and UCTE of a free semigroup action generated by proper maps for noncompact subsets. Several of their properties are provided. In Section 4 , we give some further properties of the topological entropy, LCTE and UCTE for an LCSMS. It is verified that the topological entropy, LCTE and UCTE of an LCSMS coincide with those of its one-point compactification. In Section 5 , three skew-product transformations are introduced. For a particular subset, we then give the relationship between the UCTE of a free semigroup action generated by proper maps, and the UCTE of a skew-product transformation. In Section 6, we prove that the multifractal spectrum of an LCSMS is equal to that of its one-point compactification, and it is shown that the irregular set has full UCTE of a free 
semigroup action generated by proper maps.

\section{Preliminaries}

\section{1. $\mathrm{C}-\mathrm{P}$ structure}

Let $X$ and $\mathcal{S}$ be arbitrary sets and $\mathcal{F}=\left\{U_{s}: s \in \mathcal{S}\right\}$ a collection of subsets in $X$. Following Pesin [21], we assume that there exist two functions $\eta, \psi: \mathcal{S} \rightarrow \mathbb{R}^{+}$satisfying the following conditions:

(A1) there exists $s_{0} \in \mathcal{S}$ such that $U_{s_{0}}=\emptyset$; if $U_{s}=\emptyset$ then $\eta(s)=\psi(s)=0$; if $U_{s} \neq \emptyset$ then $\eta(s)>0$ and $\psi(s)>0$.

(A2) for any $\delta>0$ one can find $\varepsilon>0$ such that $\eta(s) \leq \delta$ for any $s \in \mathcal{S}$ with $\psi(s) \leq \varepsilon$.

(A3) for any $\varepsilon>0$ there exists a finite or countable subcollection $\mathcal{G} \subset \mathcal{S}$ which covers $X$ (i.e., $\left.\bigcup_{s \in \mathcal{G}} U_{s} \supset X\right)$ and $\psi(\mathcal{G}):=\sup \{\psi(s): s \in \mathcal{G}\} \leq \varepsilon$.

Let $\xi: \mathcal{S} \rightarrow \mathbb{R}^{+}$be a function, we say that the set $\mathcal{S}$, collection of subsets $\mathcal{F}$, and the set functions $\xi, \eta, \psi$ satisfying Conditions (A1), (A2) and (A3), introduce the CarathéodoryPesin structure or C-P structure $\tau$ on $X$ and write $\tau=(\mathcal{S}, \mathcal{F}, \xi, \eta, \psi)$.

Given a subset $Z$ of $X, \alpha \in \mathbb{R}$, and $\varepsilon>0$, we define

$$
M(Z, \alpha, \varepsilon):=\inf _{\mathcal{G}}\left\{\sum_{s \in \mathcal{G}} \xi(s) \eta(s)^{\alpha}\right\},
$$

where the infimum is taken over all finite or countable subcollections $\mathcal{G} \subset \mathcal{S}$ covering $Z$ with $\psi(\mathcal{G}) \leq \varepsilon$. By Condition (A3) the function $M(Z, \alpha, \varepsilon)$ is correctly defined. It is non-decreasing as $\varepsilon$ decreases. Therefore, the following limit exists:

$$
m(Z, \alpha)=\lim _{\varepsilon \rightarrow 0} M(Z, \alpha, \varepsilon) .
$$

It was shown in 21$]$ that there exists a critical value $\alpha_{C} \in[-\infty, \infty]$ such that

$$
m(Z, \alpha)= \begin{cases}0, & \alpha>\alpha_{C} \\ \infty, & \alpha<\alpha_{C}\end{cases}
$$

We define the Carathéodory-Pesin dimension of the set $Z$ by

$$
\operatorname{dim}_{C} Z=\alpha_{C}=\inf \{\alpha: m(Z, \alpha)=0\}=\sup \{\alpha: m(Z, \alpha)=\infty\} .
$$

Then we have the following theorem.

Theorem 2.1. 21 
(1) $\operatorname{dim}_{C} \emptyset \leq 0$.

(2) $\operatorname{dim}_{C} Z_{1} \leq \operatorname{dim}_{C} Z_{2}$ if $Z_{1} \subset Z_{2} \subset X$.

(3) $\operatorname{dim}_{C}\left(\bigcup_{i \geq 0} Z_{i}\right)=\sup _{i \geq 0} \operatorname{dim}_{C} Z_{i}$, where $Z_{i} \subset X, i=0,1,2, \ldots$

Now we assume that the following condition holds:

$\left(\mathrm{A} 3^{\prime}\right)$ there exists $\epsilon>0$ such that for any $0<\varepsilon \leq \epsilon$ there exists a finite or countable subcollection $\mathcal{G} \subset \mathcal{S}$ covering $X$ such that $\psi(s)=\varepsilon$ for any $s \in \mathcal{G}$.

Given $\alpha \in \mathbb{R}$ and $\varepsilon>0$, for any subset $Z \subset X$, define

$$
R(Z, \alpha, \varepsilon)=\inf _{\mathcal{G}}\left\{\sum_{s \in \mathcal{G}} \xi(s) \eta(s)^{\alpha}\right\},
$$

where the infimum is taken over all finite or countable subcollections $\mathcal{G} \subset \mathcal{S}$ covering $Z$ such that $\psi(s)=\varepsilon$ for any $s \in \mathcal{G}$. Set

$$
\underline{r}(Z, \alpha)=\liminf _{\varepsilon \rightarrow 0} R(Z, \alpha, \varepsilon), \quad \bar{r}(Z, \alpha)=\limsup _{\varepsilon \rightarrow 0} R(Z, \alpha, \varepsilon) .
$$

It was shown in 21$]$ that there exists $\underline{\alpha}_{C}, \bar{\alpha}_{C} \in \mathbb{R}$ such that

$$
\underline{r}(Z, \alpha)=\left\{\begin{array}{ll}
\infty, & \alpha<\underline{\alpha}_{C}, \\
0, & \alpha>\underline{\alpha}_{C}
\end{array} \quad \text { and } \quad \bar{r}(Z, \alpha)= \begin{cases}\infty, & \alpha<\bar{\alpha}_{C}, \\
0, & \alpha>\bar{\alpha}_{C} .\end{cases}\right.
$$

Define the lower and upper Carathéodory-Pesin capacities of the set $Z$ by

$$
\begin{aligned}
& \underline{C a p}_{C} Z=\underline{\alpha}_{C}=\inf \{\alpha: \underline{r}(Z, \alpha)=0\}=\sup \{\alpha: \underline{r}(Z, \alpha)=\infty\}, \\
& \overline{C a p}_{C} Z=\bar{\alpha}_{C}=\inf \{\alpha: \bar{r}(Z, \alpha)=0\}=\sup \{\alpha: \bar{r}(Z, \alpha)=\infty\} .
\end{aligned}
$$

Similar to Theorem 2.1, the following theorem is provided.

Theorem 2.2. 21]

(1) $\underline{\operatorname{Cap}}_{C} \emptyset \leq 0, \overline{\operatorname{Cap}}_{C} \emptyset \leq 0$.

(2) $\underline{\operatorname{Cap}}_{C} Z_{1} \leq \underline{\operatorname{Cap}}_{C} Z_{2}$ and $\overline{\operatorname{Cap}}_{C} Z_{1} \leq \overline{\operatorname{Cap}}_{C} Z_{2}$ if $Z_{1} \subset Z_{2} \subset X$.

(3) $\underline{\operatorname{Cap}}_{C}\left(\bigcup_{i \geq 0} Z_{i}\right) \geq \sup _{i \geq 0} \underline{\operatorname{Cap}}_{C} Z_{i}, \overline{\operatorname{Cap}}_{C}\left(\bigcup_{i \geq 0} Z_{i}\right) \geq \sup _{i \geq 0} \overline{\operatorname{Cap}}_{C} Z_{i}$, where $Z_{i} \subset$ $X, i=0,1,2, \ldots$

For any $\varepsilon>0$ and subset $Z \subset X$, put

$$
\Lambda(Z, \varepsilon):=\inf _{\mathcal{G}}\left\{\sum_{s \in \mathcal{G}} \xi(s)\right\},
$$

where the infimum is taken over all finite or countable subcollections $\mathcal{G} \subset \mathcal{S}$ covering $Z$ such that $\psi(s)=\varepsilon$ for any $s \in \mathcal{G}$.

Assume that the function $\eta$ satisfies the following condition: 
(A4) $\eta\left(s_{1}\right)=\eta\left(s_{2}\right)$ for any $s_{1}, s_{2} \in \mathcal{S}$ satisfying $\psi\left(s_{1}\right)=\psi\left(s_{2}\right)$.

It was shown that the lower and upper Carathéodory-Pesin capacities admit the following description.

Theorem 2.3. 21] If the function $\eta$ satisfies Condition (A4), then for any subset $Z \subset X$,

$$
\underline{C a p}_{C} Z=\liminf _{\varepsilon \rightarrow 0} \frac{\log \Lambda(Z, \varepsilon)}{\log (1 / \eta(\varepsilon))}, \quad \overline{C a p}_{C} Z=\limsup _{\varepsilon \rightarrow 0} \frac{\log \Lambda(Z, \varepsilon)}{\log (1 / \eta(\varepsilon))} .
$$

\subsection{Words and sequences}

Denote by $F_{m}^{+}$the set of all finite words of symbols $0,1, \ldots, m-1$. For any $w \in F_{m}^{+},|w|$ stands for the length of $w$, that is, the number of symbols in $w$. We write $w^{\prime} \leq w$ if there exists a word $w^{\prime \prime} \in F_{m}^{+}$such that $w=w^{\prime \prime} w^{\prime}$. For $w=i_{1} \cdots i_{k} \in F_{m}^{+}$, denote $\bar{w}=i_{k} \cdots i_{1}$.

Denote by $\Sigma_{m}$ the set of all two-side infinite sequences of symbols $0,1, \ldots, m-1$, that is,

$$
\Sigma_{m}=\left\{\omega=\left(\ldots, \omega_{-1}, \omega_{0}, \omega_{1}, \ldots\right) \mid \omega_{i}=0,1, \ldots, m-1 \text { for all integer } i\right\}
$$

A metric on $\Sigma_{m}$ is introduced by setting

$$
d^{\prime}\left(\omega, \omega^{\prime}\right)=\frac{1}{2^{k}}, \quad \text { where } k=\inf \left\{|n|: \omega_{n} \neq \omega_{n}^{\prime}\right\}
$$

Obviously, $\Sigma_{m}$ is compact with respect to this metric. The Bernoulli shift $\sigma_{m}: \Sigma_{m} \rightarrow$ $\Sigma_{m}$ is a homeomorphism of $\Sigma_{m}$ given by the formula

$$
\left(\sigma_{m} \omega\right)_{i}=\omega_{i+1}
$$

Assume that $\omega \in \Sigma_{m}, w \in F_{m}^{+}, a, b$ are integers, and $a \leq b$. We write $\left.\omega\right|_{[a, b]}=w$ if $w=\omega_{a} \omega_{a+1} \cdots \omega_{b-1} \omega_{b}$.

Let $\Sigma_{m}^{+}$be the set of all one-side infinite sequences of symbols $0,1, \ldots, m-1$ :

$$
\Sigma_{m}^{+}=\left\{\omega=\left(i_{0}, i_{1}, \ldots\right): i_{j}=0,1, \ldots, m-1 \text { for all integer } j\right\}
$$

\subsection{Proper map and admissible metric}

Let $X$ be a topological space and $f: X \rightarrow X$ be a proper map, i.e., $f$ is a continuous map such that the pre-image by $f$ of any compact set is compact. A finite open cover $\mathcal{U}$ of $X$ is called admissible cover if for each $A \in \mathcal{U}$, the closure or the complement of $A$ is compact.

If $X$ is an LCSMS, we can associate $X$ with its one-point compactification, which is denoted by $\tilde{X}$. We know that $\widetilde{X}$ is defined as the disjoint union of $X$ with $\{\infty\}$, where $\infty$ is some point not in $X$ called the point at infinity. The topology in $\tilde{X}$ consist of the 
former open sets in $X$ and the sets $A \cup\{\infty\}$, where the complement of $A$ in $X$ is compact. Let $f: X \rightarrow X$ be a proper map. Defining $\tilde{f}: \widetilde{X} \rightarrow \widetilde{X}$ by

$$
\widetilde{f}(\widetilde{x})= \begin{cases}f(\widetilde{x}), & \widetilde{x} \neq \infty \\ \infty, & \widetilde{x}=\infty\end{cases}
$$

It is easy to see that $\tilde{f}$ is also a proper map, which is called the extension of $f$ to $\tilde{X}$. Note that the separability of $X$ is equivalent to the metrizability of $\widetilde{X}$.

Let $(X, d)$ be a metric space and $B(x, \delta)$ represents the open ball centered at $x$ with radius $\delta>0$. The metric $d$ is called admissible [20] if the following conditions are satisfied:

(1) If $\alpha_{\delta}=\left\{B\left(x_{1}, \delta\right), \ldots, B\left(x_{k}, \delta\right)\right\}$ is a cover of $X$, for every $\delta \in(a, b)$, where $0<a<b$, then there exists $\delta_{\varepsilon} \in(a, b)$ such that $\alpha_{\delta_{\varepsilon}}$ is admissible.

(2) Every admissible cover of $X$ has a Lebesgue number.

Obviously, if $(X, d)$ is compact, then $d$ is automatically admissible.

3. Topological entropy, LCTE, UCTE and their properties

In this section, by using the $\mathrm{C}-\mathrm{P}$ structure, we give the notions of the topological entropy, LCTE and UCTE of a free semigroup action generated by proper maps for noncompact subsets. Such works extend the previous notions defined by Ju et al. [13, Ma et al. [17], Patrão [20] and Tian et al. [24]. Moreover, some basic properties of these entropies are provided.

\subsection{Notions of the topological entropy, LCTE and UCTE}

Let $X$ be a topological space, $f_{i}: X \rightarrow X(i=0,1,2, \ldots, m-1)$ be proper maps and $\mathcal{U}$ be an admissible cover of $X$. Suppose that $G$ is the free semigroup acting on $X$ generated by $\left\{f_{0}, f_{1}, \ldots, f_{m-1}\right\}$. Consider a finite open cover $\mathcal{U}$ of $X$, let

$$
\mathcal{S}_{n+1}(\mathcal{U}):=\left\{\mathbf{U}=\left(U_{0}, U_{1}, \ldots, U_{n}\right): U_{i} \in \mathcal{U}, i=0,1, \ldots, n\right\}
$$

For any string $\mathbf{U} \in \mathcal{S}_{n+1}(\mathcal{U})$, define the length of $\mathbf{U}$ to be $L(\mathbf{U}):=n+1$. We put $S=S(\mathcal{U})=\bigcup_{n>0} S_{n}(\mathcal{U})$. For any $\omega=\left(i_{1}, i_{2}, \ldots, i_{n}, \ldots\right) \in \Sigma_{m}^{+}, n \geq 1$, and a given string $\mathbf{U}=\left(U_{0}, U_{1}, \ldots, U_{n}\right) \in \mathcal{S}_{n+1}(\mathcal{U})$, we associate the set

$$
X_{\omega}(\mathbf{U})=\left\{x \in X: x \in U_{0}, f_{i_{j}} \circ \cdots \circ f_{i_{1}}(x) \in U_{j}, j=1,2, \ldots, n\right\} .
$$

If $w=i_{1} i_{2} \cdots i_{n}=\left.\omega\right|_{[0, n-1]} \in F_{m}^{+}$, we also denote $X_{\omega}(\mathbf{U})$ by $X_{w}(\mathbf{U})$ for convenience. Define

$$
\mathcal{F}=\left\{X_{\omega}(\mathbf{U}): \mathbf{U} \in \mathcal{S}(\mathcal{U}) \text { and } \omega \in \Sigma_{m}^{+}\right\}
$$


and three functions $\xi, \eta, \psi: \mathcal{S} \rightarrow \mathbb{R}^{+}$as follows:

$$
\xi(\mathbf{U}) \equiv 1, \quad \eta(\mathbf{U})=\exp (-L(\mathbf{U})), \quad \psi(\mathbf{U})=L(\mathbf{U})^{-1}
$$

It is easy to see that the set $\mathcal{S}$, collection of subsets $\mathcal{F}$, and the functions $\xi, \eta$ and $\psi$ determine a $\mathrm{C}-\mathrm{P}$ structure $\tau=(\mathcal{S}, \mathcal{F}, \xi, \eta, \psi)$ on $X$. Given $w \in F_{m}^{+},|w|=N, Z \subset X$ and $\alpha \geq 0$, we define

$$
M_{w}(Z, \alpha, \mathcal{U}, N):=\inf _{\mathcal{G}_{w}}\left\{\sum_{\mathbf{U} \in \mathcal{G}_{w}} \exp (-\alpha L(\mathbf{U}))\right\},
$$

where the infimum is taken over all finite or countable collections of strings $\mathcal{G}_{w} \subset \mathcal{S}(\mathcal{U})$ such that $L(\mathbf{U}) \geq N+1$ for all $\mathbf{U} \in \mathcal{G}_{w}$ and $\mathcal{G}_{w}$ covers $Z$ (i.e., for any $\mathbf{U} \in \mathcal{G}_{w}$, there exists $\omega_{\mathbf{U}} \in \Sigma_{m}^{+}$such that $\left.\omega_{\mathbf{U}}\right|_{[0, N-1]}=w$ and $\bigcup_{\mathbf{U} \in \mathcal{G}_{w}} X_{\omega_{\mathbf{U}}}(\mathbf{U}) \supset Z$, in other words, for any $\mathbf{U} \in \mathcal{G}_{w}$, there is $w_{\mathbf{U}} \in F_{m}^{+}$such that $\bar{w} \leq \overline{w_{\mathbf{U}}}$ and $\left.\bigcup_{\mathbf{U} \in \mathcal{G}_{w}} X_{w_{\mathbf{U}}}(\mathbf{U}) \supset Z\right)$.

Let

$$
M(Z, \alpha, \mathcal{U}, N)=\frac{1}{m^{N}} \sum_{|w|=N} M_{w}(Z, \alpha, \mathcal{U}, N) .
$$

We can easily verify that the function $M(Z, \alpha, \mathcal{U}, N)$ is non-decreasing as $N$ increases. Therefore the following limit exists

$$
m(Z, \alpha, \mathcal{U})=\lim _{N \rightarrow \infty} M(Z, \alpha, \mathcal{U}, N)
$$

Furthermore, given $w \in F_{m}^{+}$and $|w|=N$, by the Condition $\left(\mathrm{A} 3^{\prime}\right)$ in Section 2.1, we can define

$$
R_{w}(Z, \alpha, \mathcal{U}, N)=\inf _{\mathcal{G}_{w}}\left\{\sum_{\mathbf{U} \in \mathcal{G}_{w}} \exp (-\alpha(N+1))\right\}=\Lambda_{w}(Z, \mathcal{U}, N) \exp (-\alpha(N+1))
$$

where $\Lambda_{w}(Z, \mathcal{U}, N)=\inf _{\mathcal{G}_{w}}\left\{\operatorname{card}\left(\mathcal{G}_{w}\right)\right\}$, the infimum is taken over all finite or countable collections of strings $\mathcal{G}_{w} \subset \mathcal{S}(\mathcal{U})$ such that $L(\mathbf{U})=N+1$ for all $\mathbf{U} \in \mathcal{G}_{w}$ and $\mathcal{G}_{w}$ covers $Z$ (i.e., $\bigcup_{\mathbf{U} \in \mathcal{G}_{w}} X_{w}(\mathbf{U}) \supset Z$ ).

Let

$$
R(Z, \alpha, \mathcal{U}, N)=\frac{1}{m^{N}} \sum_{|w|=N} R_{w}(Z, \alpha, \mathcal{U}, N)
$$

and

$$
\Lambda(Z, \mathcal{U}, N)=\frac{1}{m^{N}} \sum_{|w|=N} \Lambda_{w}(Z, \mathcal{U}, N)
$$

Then we have

$$
R(Z, \alpha, \mathcal{U}, N)=\Lambda(Z, \mathcal{U}, N) \exp (-\alpha(N+1))
$$


Set

$$
\underline{r}(Z, \alpha, \mathcal{U})=\liminf _{N \rightarrow \infty} R(Z, \alpha, \mathcal{U}, N) \quad \text { and } \quad \bar{r}(Z, \alpha, \mathcal{U})=\limsup _{N \rightarrow \infty} R(Z, \alpha, \mathcal{U}, N) .
$$

By the definition of $\mathrm{C}-\mathrm{P}$ structure,we can define

$$
\begin{aligned}
h_{Z}(\mathcal{U}, G) & =\inf \{\alpha: m(Z, \alpha, \mathcal{U})=0\}=\sup \{\alpha: m(Z, \alpha, \mathcal{U})=\infty\}, \\
\underline{C h}_{Z}(\mathcal{U}, G) & =\inf \{\alpha: \underline{r}(Z, \alpha, \mathcal{U})=0\}=\sup \{\alpha: \underline{r}(Z, \alpha, \mathcal{U})=\infty\}, \\
\overline{C h}_{Z}(\mathcal{U}, G) & =\inf \{\alpha: \bar{r}(Z, \alpha, \mathcal{U})=0\}=\sup \{\alpha: \bar{r}(Z, \alpha, \mathcal{U})=\infty\} .
\end{aligned}
$$

Moreover, define

$$
h_{Z}(G):=\sup _{\mathcal{U}} h_{Z}(\mathcal{U}, G), \quad \underline{C h}_{Z}(G):=\sup _{\mathcal{U}} \underline{C h} Z(\mathcal{U}, G), \quad \overline{C h}_{Z}(G):=\sup _{\mathcal{U}} \overline{C h}_{Z}(\mathcal{U}, G),
$$

where the supremum is taken over all admissible covers of $X$. We call the quantities $h_{Z}(G), \underline{C h}_{Z}(G)$ and $\overline{C h}_{Z}(G)$, respectively the topological entropy and lower and upper capacity topological entropy of $G$ with respect to $Z$.

Remark 3.1. (1) By the definition, $h_{Z}(G) \leq \underline{C h}_{Z}(G) \leq \overline{C h}_{Z}(G), \forall Z \subset X$.

(2) If $X$ is a compact metric space, the topological entropy, LCTE and UCTE in this paper coincide with those defined by Ju et al. 13 .

(3) If $m=1$, i.e., $G$ is generated by $\{f\}$, then $h_{Z}(G)=h_{Z}(f), \underline{C h}_{Z}(G)=\underline{C h}_{Z}(f)$, $\overline{C h}_{Z}(G)=\overline{C h}_{Z}(f)$, where $h_{Z}(f), \underline{C h}_{Z}(f)$ and $\overline{C h}_{Z}(f)$ are the topological entropy, LCTE and UCTE defined by Ma et al. [17. If $m>1$, there are several proper maps acting on $X$, so each point has many orbits, which makes the system more complex and challenges us in describing the complexity of the system. Indeed, $M(\cdot, \cdot, \cdot, \cdot)$ in 3.1 and $R(\cdot, \cdot, \cdot, \cdot)$ in 3.2 have a different denominator $m^{N}$ which is equal to the number of orbits of length $N$ at a point, so $M(\cdot, \cdot, \cdot, \cdot)$ and $R(\cdot, \cdot, \cdot, \cdot)$ can be regarded as orbital averages.

\subsection{Some properties of the topological entropy, LCTE and UCTE}

Using the basic properties of the $\mathrm{C}-\mathrm{P}$ structure, we give the following three properties. Since they are special cases of Theorems 2.1, 2.2, 2.3 respectively, we omit their proofs.

Proposition 3.2. (1) $h_{\emptyset}(G)=0$.

(2) $h_{Z_{1}}(G) \leq h_{Z_{2}}(G)$ if $Z_{1} \subset Z_{2}$.

(3) $h_{Z}(G)=\sup _{i \geq 1} h_{Z_{i}}(G)$, where $Z=\bigcup_{i \geq 1} Z_{i}$ and $Z_{i} \subset X, i=1,2, \ldots$

Proposition 3.3. (1) $C h_{\emptyset}(G)=\overline{C h_{\emptyset}}(G)=0$.

(2) $\underline{C h}_{Z_{1}}(G) \leq \underline{C h}_{Z_{2}}(G)$ and $\overline{C h}_{Z_{1}}(G) \leq \overline{C h}_{Z_{2}}(G)$ if $Z_{1} \subset Z_{2}$. 
(3) $\underline{C h}_{Z}(G) \geq \sup _{i \geq 1} \underline{C h}_{Z_{i}}(G)$ and $\overline{C h}_{Z}(G) \geq \sup _{i \geq 1} \overline{C h}_{Z_{i}}(G)$, where $Z=\bigcup_{i \geq 1} Z_{i}$ and $Z_{i} \subset X, i=1,2, \ldots$

Proposition 3.4. For any admissible cover $\mathcal{U}$ of $X$ and any set $Z \subset X$, we have

$$
\underline{C h}_{Z}(\mathcal{U}, G)=\liminf _{N \rightarrow \infty} \frac{\log \Lambda(Z, \mathcal{U}, N)}{N}, \quad \overline{C h}_{Z}(\mathcal{U}, G)=\limsup _{N \rightarrow \infty} \frac{\log \Lambda(Z, \mathcal{U}, N)}{N} .
$$

Remark 3.5. (1) By Proposition 3.4, we get that

$$
\underline{C h_{Z}}(G)=\sup _{\mathcal{U}} \liminf _{N \rightarrow \infty} \frac{\log \Lambda(Z, \mathcal{U}, N)}{N}, \quad \overline{C h}_{Z}(G)=\sup _{\mathcal{U}} \limsup _{N \rightarrow \infty} \frac{\log \Lambda(Z, \mathcal{U}, N)}{N} .
$$

(2) It is easy to see that $\overline{C h}_{X}(G)=h\left(f_{0}, \ldots, f_{m-1}\right)$, where $h\left(f_{0}, \ldots, f_{m-1}\right)$ denotes the topological entropy of a free semigroup action in $[24$.

Moreover, we can prove the following proposition.

Proposition 3.6. Let $X_{1}, X_{2}$ be topological spaces, $g_{i}: X_{1} \rightarrow X_{1}, f_{i}: X_{2} \rightarrow X_{2}(i=$ $0,1,2, \ldots, m-1)$ be proper maps. Suppose that $G_{1}$ and $G_{2}$ are the free semigroups acting on $X_{1}$ and $X_{2}$, generated by $\left\{g_{0}, g_{1}, \ldots, g_{m-1}\right\}$ and $\left\{f_{0}, f_{1}, \ldots, f_{m-1}\right\}$ respectively. There exists a proper surjective map $\pi: X_{1} \rightarrow X_{2}$ such that $\pi \circ g_{i}=f_{i} \circ \pi(i=0,1, \ldots, m-1)$, then for any $Z \subset X$, we have

$$
h_{Z}\left(G_{1}\right) \geq h_{\pi Z}\left(G_{2}\right), \quad \underline{C h}_{Z}\left(G_{1}\right) \geq \underline{C h}_{\pi Z}\left(G_{2}\right) \quad \text { and } \quad \overline{C h}_{Z}\left(G_{1}\right) \geq \overline{C h}_{\pi Z}\left(G_{2}\right) .
$$

Specifically, if $\left(X_{1}, g_{i}\right)$ and $\left(X_{2}, f_{i}\right)$ topologically conjugate $(i=0,1, \ldots, m-1)$, i.e., $\pi$ is a homeomorphism, then

$$
h_{Z}\left(G_{1}\right)=h_{\pi Z}\left(G_{2}\right), \quad \underline{C h}_{Z}\left(G_{1}\right)=\underline{C h}_{\pi Z}\left(G_{2}\right) \quad \text { and } \quad \overline{C h}_{Z}\left(G_{1}\right)=\overline{C h}_{\pi Z}\left(G_{2}\right) .
$$

Proof. Let $\mathcal{U}_{2}$ be an admissible cover of $X_{2}$. Set

$$
\mathcal{U}_{1}=\left\{\pi^{-1} U_{i}: U_{i} \in \mathcal{U}_{2}\right\}
$$

Then $\mathcal{U}_{1}$ is an admissible cover of $X_{1}$. For each string $\mathbf{U}=\left(U_{0}, U_{1}, \ldots, U_{N}\right) \in \mathcal{S}\left(\mathcal{U}_{2}\right)$, let $\pi^{-1} \mathbf{U}=\left(\pi^{-1} U_{0}, \pi^{-1} U_{1}, \ldots, \pi^{-1} U_{N}\right)$, then $\pi^{-1} \mathbf{U} \in \mathcal{S}\left(\mathcal{U}_{1}\right)$. On the other hand, for each string $\mathbf{V} \in \mathcal{S}\left(\mathcal{U}_{1}\right)$, there is a unique string $\mathbf{U} \in \mathcal{S}\left(\mathcal{U}_{2}\right)$ such that $\mathbf{V}=\pi^{-1} \mathbf{U}$. We note that for any $w \in F_{m}^{+}$with $|w|=N$,

$$
L(\mathbf{U})=L\left(\pi^{-1} \mathbf{U}\right)=L(\mathbf{V})
$$

By the commutativity,

$$
X_{w}(\mathbf{V})=X_{w}\left(\pi^{-1} \mathbf{U}\right)=\pi^{-1} X_{w}(\mathbf{U})
$$


Thus

$$
M_{w}\left(\pi Z, \alpha, \mathcal{U}_{2}, N\right)=M_{w}\left(Z, \alpha, \mathcal{U}_{1}, N\right)
$$

Then

$$
M\left(\pi Z, \alpha, \mathcal{U}_{2}, N\right)=M\left(Z, \alpha, \mathcal{U}_{1}, N\right)
$$

and

$$
m\left(\pi Z, \alpha, \mathcal{U}_{2}\right)=m\left(Z, \alpha, \mathcal{U}_{1}\right)
$$

Moreover,

$$
h_{\pi Z}\left(G_{2}, \mathcal{U}_{2}\right)=h_{Z}\left(G_{1}, \mathcal{U}_{1}\right) \leq h_{Z}\left(G_{1}\right) .
$$

According to the arbitrarily of $\mathcal{U}_{2}$,

$$
h_{\pi Z}\left(G_{2}\right) \leq h_{Z}\left(G_{1}\right) .
$$

If $\pi$ is a homeomorphism, $\pi^{-1}$ is a continuous surjection from $X_{2}$ to $X_{1}$, by the above proof we have

$$
h_{Z}\left(G_{1}\right) \leq h_{\pi Z}\left(G_{2}\right)
$$

Therefore,

$$
h_{Z}\left(G_{1}\right)=h_{\pi Z}\left(G_{2}\right) .
$$

The others can be obtained in a similar fashion.

Based on Remark 3.1 (2), we can use the analogous method as that of [13, Theorems 3.3 and 3.6] to prove the following two propositions, so we omit their proofs.

Proposition 3.7. For any $G$-invariant set $Z \subset X$ (i.e., $f_{i}^{-1}(Z)=Z$ for all $f_{i} \in G$ ), we have

$$
\underline{C h}_{Z}(G)=\overline{C h}_{Z}(G) \text {. }
$$

Proposition 3.8. Under Condition 3.5 of [13], for any compact $G$-invariant set $Z \subset X$, we have

$$
h_{Z}(G)=\underline{C h}_{Z}(G)=\overline{C h}_{Z}(G) .
$$

Example 3.9. Let $(X, d)$ be a metric space, and $f_{0}, f_{1}, \ldots, f_{m-1}$ be uniformly continuous transformations from $X$ to itself. Suppose that $G$ is the free semigroup acting on $X$ generated by $\left\{f_{0}, f_{1}, \ldots, f_{m-1}\right\}$. If the family $G$ are equicontinuous, then

$$
\underline{C h}_{Z}(G)=\overline{C h}_{Z}(G)=h_{Z}(G)=0, \quad \forall Z \subset X .
$$

Proof. It was shown in 24 that

$$
\overline{C h}_{X}(G)=0 .
$$

According to Remark 3.1(1) and Proposition 3.3(2), we have

$$
\underline{C h}_{Z}(G)=\overline{C h}_{Z}(G)=h_{Z}(G)=0, \quad \forall Z \subset X .
$$




\section{Topological entropy, LCTE and UCTE of an LCSMS}

In this section, we assume that $X$ is an LCSMS, thus it can be associated with its one-point compactification $\tilde{X}$, which makes us better to study further properties of the topological entropy, LCTE and UCTE in this paper. The following theorem describes the relations of the entropies between two dynamical systems.

Theorem 4.1. Let $X$ be an LCSMS. Suppose that $G$ is the free semigroup acting on $X$ generated by $\left\{f_{0}, f_{1}, \ldots, f_{m-1}\right\}$, where $f_{i}: X \rightarrow X(i=0,1, \ldots, m-1)$ are proper maps. Let $d$ be the metric given by the restriction to $X$ of some metric $\widetilde{d}$ on $\tilde{X}$. Then for any $Z \subset X$, the following limits exist:

$$
\lim _{|\mathcal{U}| \rightarrow 0} h_{Z}(\mathcal{U}, G)=h_{Z}(G), \quad \lim _{|\mathcal{U}| \rightarrow 0} \underline{C h_{Z}}(\mathcal{U}, G)=\underline{C h_{Z}}(G), \quad \lim _{|\mathcal{U}| \rightarrow 0} \overline{C h}_{Z}(\mathcal{U}, G)=\overline{C h}_{Z}(G),
$$

where $\mathcal{U}$ is any admissible cover of $X$.

Lemma 4.2. 20 Let $X$ be an LCSMS, $f: X \rightarrow X$ be a proper map. Let $d$ be the metric given by the restriction to $X$ of some metric $\widetilde{d}$ on $\widetilde{X}$, the one-point compactification of $X$. Then $d$ is an admissible metric and for any $\varepsilon>0$ there exists an admissible cover of $X$, such that the diameter of this cover is less than $\varepsilon$.

Proof of Theorem 4.1. We just need to prove that

$$
\varliminf_{|\mathcal{U}| \rightarrow 0} h_{Z}(\mathcal{U}, G) \geq h_{Z}(G)
$$

By Lemma 4.2, for any $\varepsilon>0$, there exists an admissible cover $\mathcal{V}$ such that $|\mathcal{V}|<\varepsilon$ and $\sup _{\mathcal{U}} h_{Z}(\mathcal{U}, G)-\varepsilon \leq h_{Z}(\mathcal{V}, G)$. Let $\delta$ be a Lebesgue number of $\mathcal{V}$ and $\mathcal{W}$ be an admissible cover of $X$ with $|\mathcal{W}|<\delta$. Then each element of $\mathcal{W}$ is contained in some element of $\mathcal{V}$. Thus for any $w \in F_{m}^{+}$with $|w|=N$, we have

$$
M_{w}(Z, \alpha, \mathcal{V}, N) \leq M_{w}(Z, \alpha, \mathcal{W}, N)
$$

Then

$$
M(Z, \alpha, \mathcal{V}, N) \leq M(Z, \alpha, \mathcal{W}, N)
$$

Letting $N \rightarrow \infty$, we have

$$
m(Z, \alpha, \mathcal{V}) \leq m(Z, \alpha, \mathcal{W})
$$

Thus,

$$
h_{Z}(\mathcal{V}, G) \leq h_{Z}(\mathcal{W}, G)
$$

Moreover,

$$
\sup _{\mathcal{U}} h_{Z}(\mathcal{U}, G)-\varepsilon \leq h_{Z}(\mathcal{V}, G) \leq h_{Z}(\mathcal{W}, G)
$$


Letting $|\mathcal{W}| \rightarrow 0$, we get that

$$
\sup _{\mathcal{U}} h_{Z}(\mathcal{U}, G)-\varepsilon \leq \underline{\lim }_{|\mathcal{W}| \rightarrow 0} h_{Z}(\mathcal{W}, G)
$$

According to the arbitrarily of $\varepsilon$,

$$
\sup _{\mathcal{U}} h_{Z}(\mathcal{U}, G) \leq \varliminf_{\mid \underline{\mathcal{W} \mid \rightarrow 0}} h_{Z}(\mathcal{W}, G) .
$$

Therefore,

$$
\lim _{|\mathcal{U}| \rightarrow 0} h_{Z}(\mathcal{U}, G)=h_{Z}(G) .
$$

The existence of the two other limits can be proved in similar ways.

Remark 4.3. It is easy to see that the topological entropy is a generalization of the topological entropy defined by Ju et al. [13].

Theorem 4.4. Let $X$ be an LCSMS, $f_{i}: X \rightarrow X(i=0,1, \ldots, m-1)$ be proper maps and $\tilde{f}_{i}(i=0,1, \ldots, m-1)$ be its extension of $f_{i}$ to $\widetilde{X}$. Suppose that $G$ and $\widetilde{G}$ are the free semigroups acting on $X$ and $\widetilde{X}$ respectively, generated by $\left\{f_{0}, f_{1}, \ldots, f_{m-1}\right\}$ and $\left\{\widetilde{f}_{0}, \tilde{f}_{1}, \ldots, \widetilde{f}_{m-1}\right\}$ respectively. Then for any $Z \subset X$,

$$
h_{Z}(G)=h_{Z}(\widetilde{G}), \quad \underline{C h}_{Z}(G)=\underline{C h}_{Z}(\widetilde{G}) \quad \text { and } \quad \overline{C h}_{Z}(G)=\overline{C h}_{Z}(\widetilde{G})
$$

Proof. Let $d$ be the metric given by the restriction to $X$ of some metric $\widetilde{d}$ on $\widetilde{X}$ and $\mathcal{U}=\left\{U_{0}, U_{1}, \ldots, U_{k-1}\right\}$ be an admissible cover of $X$. Let $\widetilde{U}_{i}=\left\{y \in \widetilde{X}: \exists x \in U_{i}, \widetilde{d}(x, y)<\right.$ $\left.\left|U_{i}\right|\right\}(0 \leq i \leq k-1)$, then $\widetilde{\mathcal{U}}=\left\{\widetilde{U}_{0}, \ldots, \widetilde{U}_{k-1}\right\}$ is an open cover of $\widetilde{X}$ and $|\mathcal{U}| \rightarrow 0$ implies $|\widetilde{\mathcal{U}}| \rightarrow 0$. Given $w \in F_{m}^{+}$with $|w|=N$, assume $\mathcal{G}_{w} \subset \bigcup_{n \geq N} S_{n}(\mathcal{U})$ covers $Z$. For any $\mathbf{U}=\left(U_{i_{0}}, U_{i_{1}}, \ldots, U_{i_{n-1}}\right) \in \mathcal{G}_{w}$, put $\widetilde{\mathbf{U}}=\left(\widetilde{U}_{i_{0}}, \widetilde{U}_{i_{1}}, \ldots, \widetilde{U}_{i_{n-1}}\right) \in S(\widetilde{\mathcal{U}})$ and denote by $\widetilde{\mathcal{G}}_{w}$ the collection of all these strings, then $\widetilde{\mathcal{G}}_{w}$ covers $Z \subset \widetilde{X}$. Hence,

$$
\begin{aligned}
M_{w}(Z, \alpha, \mathcal{U}, N) & =\inf _{\mathcal{G}_{w}}\left\{\sum_{\mathbf{U} \in \mathcal{G}_{w}} \exp (-\alpha L(\mathbf{U}))\right\}=\inf _{\widetilde{\mathcal{G}}_{w}}\left\{\sum_{\widetilde{\mathbf{U}} \in \widetilde{\mathcal{G}}_{w}} \exp (-\alpha L(\widetilde{\mathbf{U}}))\right\} \\
& \geq \inf _{\mathcal{G}_{w}^{\prime}}\left\{\sum_{\mathbf{U}^{\prime} \in \mathcal{G}_{w}^{\prime}} \exp \left(-\alpha L\left(\mathbf{U}^{\prime}\right)\right)\right\}:=\widetilde{M}_{w}(Z, \alpha, \widetilde{\mathcal{U}}, N)
\end{aligned}
$$

where the last infimum is taken over all finite or countable collections of strings $\mathcal{G}_{w}^{\prime} \subset S(\widetilde{\mathcal{U}})$ that covers $Z$. Then we have

$$
M(Z, \alpha, \mathcal{U}, N) \geq M(Z, \alpha, \widetilde{\mathcal{U}}, N)
$$

Letting $N \rightarrow \infty$, we get

$$
m(Z, \alpha, \mathcal{U}) \geq m(Z, \alpha, \widetilde{\mathcal{U}})
$$


Moreover,

$$
h_{Z}(\mathcal{U}, G) \geq h_{Z}(\widetilde{\mathcal{U}}, \widetilde{G})
$$

Applying Theorem 4.1 and letting $|\mathcal{U}| \rightarrow 0$, it follows that

$$
h_{Z}(G) \geq h_{Z}(\widetilde{G}) .
$$

On the other hand, let $\widetilde{\mathcal{U}}_{\varepsilon / 2}=\left\{\widetilde{B}\left(\widetilde{x}_{0}, \varepsilon / 2\right), \ldots, \widetilde{B}\left(\widetilde{x}_{k-1}, \varepsilon / 2\right)\right\}$ be a cover of $\widetilde{X}$, for any $\varepsilon \in(a, b)$, where $0<a<b$. Since $X$ is dense in $\tilde{X}$, there exist $\left\{x_{0}, \ldots, x_{k-1}\right\} \subset X$, such that $\widetilde{d}\left(x_{i}, \widetilde{x}_{i}\right)<\varepsilon / 2(0 \leq i \leq k-1)$. For any $x \in X$, there is $\widetilde{x}_{i} \in\left\{\widetilde{x}_{0}, \ldots, \widetilde{x}_{k-1}\right\}$, such that, $\widetilde{d}\left(x, \widetilde{x}_{i}\right)<\varepsilon / 2$. Then we have

$$
d\left(x, x_{i}\right) \leq \widetilde{d}\left(x, \widetilde{x}_{i}\right)+\widetilde{d}\left(x_{i}, \widetilde{x}_{i}\right)<\varepsilon
$$

showing that $\left\{B\left(x_{0}, \varepsilon\right), \ldots, B\left(x_{k-1}, \varepsilon\right)\right\}$ is a cover of $X$. By Lemma 4.2 , $d$ is an admissible metric, then there exists $\delta \in(a, b)$ such that $\mathcal{U}_{\delta}=\left\{B\left(x_{0}, \delta\right), \ldots, B\left(x_{k-1}, \delta\right)\right\}$ is an admissible cover of $X$. Let $a<\varepsilon_{1}<\delta<b$, given $w \in F_{m}^{+}$with $|w|=N$. Assume $\widetilde{\mathcal{G}}_{w} \subset \bigcup_{n \geq N} S_{n}\left(\widetilde{\mathcal{U}}_{\varepsilon_{1} / 2}\right)$ covers $Z$. For any $\widetilde{\mathbf{U}}=\left\{\widetilde{B}\left(\widetilde{x}_{i_{0}}, \varepsilon_{1} / 2\right), \ldots, \widetilde{B}\left(\widetilde{x}_{i_{p}}, \varepsilon_{1} / 2\right)\right\} \in \widetilde{\mathcal{G}}_{w}$, put $\mathbf{U}=\left\{B\left(x_{i_{0}}, \delta\right), \ldots, B\left(x_{i_{p}}, \delta\right)\right\} \in S\left(\mathcal{U}_{\delta}\right)$ and denote by $\mathcal{G}_{w}$ the collection of all these strings, then we can easily verify that $\mathcal{G}_{w}$ covers $Z$. It follows that

$$
\begin{aligned}
\widetilde{M}_{w}\left(Z, \alpha, \widetilde{\mathcal{U}}_{\varepsilon_{1} / 2}, N\right) & =\inf _{\widetilde{\mathcal{G}}_{w}}\left\{\sum_{\widetilde{\mathbf{U}} \in \widetilde{\mathcal{G}}_{w}} \exp (-\alpha L(\widetilde{\mathbf{U}}))\right\}=\inf _{\mathcal{G}_{w}}\left\{\sum_{\mathbf{U} \in \mathcal{G}_{w}} \exp (-\alpha L(\mathbf{U}))\right\} \\
& \geq \inf _{\mathcal{G}_{w}^{\prime}}\left\{\sum_{\mathbf{U}^{\prime} \in \mathcal{G}_{w}^{\prime}} \exp \left(-\alpha L\left(\mathbf{U}^{\prime}\right)\right)\right\}=M_{w}\left(Z, \alpha, \mathcal{U}_{\delta}, N\right),
\end{aligned}
$$

where the last infimum is taken over all finite or countable collections of strings $\mathcal{G}_{w}^{\prime} \subset S\left(\mathcal{U}_{\delta}\right)$ that covers $Z$. Moreover,

$$
h_{Z}\left(\widetilde{\mathcal{U}}_{\varepsilon_{1} / 2}, \widetilde{G}\right) \geq h_{Z}\left(\mathcal{U}_{\delta}, G\right) .
$$

Letting $b \rightarrow 0$, then $\varepsilon_{1} / 2 \rightarrow 0$ and $\delta \rightarrow 0$, thus we get

$$
h_{Z}(\widetilde{G}) \geq h_{Z}(G)
$$

Therefore,

$$
h_{Z}(\widetilde{G})=h_{Z}(G) .
$$

The other two equalities can be obtained in a similar fashion.

Remark 4.5. It was shown in $\left[17\right.$ that $h_{Z}(\widetilde{f})=h_{Z}(f), \overline{C h}_{Z}(\widetilde{f})=\overline{C h}_{Z}(f)$ and $\underline{C h}_{Z}(\widetilde{f})=$ $\mathrm{Ch}_{Z}(f)$, this is a special case of Theorem 4.4. 
Let $X$ be a metric space with metric $d, f_{i}: X \rightarrow X(i=0,1, \ldots, m-1)$ be proper maps. Let $w=i_{1} i_{2} \cdots i_{k} \in F_{m}^{+}$, where $i_{j}=0,1, \ldots, m-1$ for all $j=1, \ldots, k$. Let $f_{w}=f_{i_{1}} f_{i_{2}} \cdots f_{i_{k}}, f_{w}^{-1}=f_{i_{k}}^{-1} f_{i_{k-1}}^{-1} \cdots f_{i_{1}}^{-1}$. Obviously, $f_{w w^{\prime}}=f_{w} f_{w^{\prime}}$.

To each $w \in F_{m}^{+}$, a new metric $d_{w}$ on $X$ (named Bowen metric) is given by

$$
d_{w}\left(x_{1}, x_{2}\right)=\max _{w^{\prime} \leq \bar{w}} d\left(f_{w^{\prime}}\left(x_{1}\right), f_{w^{\prime}}\left(x_{2}\right)\right)
$$

Fix a number $\delta>0$, given $w \in F_{m}^{+}$and a point $x \in X$, define the $(w, \delta)$-Bowen ball at $x$ by

$$
B_{w}(x, \delta)=\left\{y \in X: d_{w}(x, y) \leq \delta\right\}
$$

For any subset $Z \subset X, w \in F_{m}^{+}$and $\varepsilon>0$, a subset $E \subset X$ is said to be a $\left(w, \varepsilon, Z, f_{0}, \ldots\right.$, $\left.f_{m-1}\right)$-spanning set of $Z[26]$, if for any $x \in Z$, there exists $y \in E$ such that $d_{w}(x, y)<\varepsilon$. Let $B\left(w, \varepsilon, Z, f_{0}, \ldots, f_{m-1}\right)$ denotes the minimum cardinality of any $\left(w, \varepsilon, Z, f_{0}, \ldots, f_{m-1}\right)$ spanning sets of $Z$.

A subset $F \subset Z$ is said to be a $\left(w, \varepsilon, Z, f_{0}, \ldots, f_{m-1}\right)$-separated set of $Z$, if $x, y \in F$, $x \neq y$ implies $d_{w}(x, y) \geq \varepsilon$. Let $N\left(w, \varepsilon, Z, f_{0}, \ldots, f_{m-1}\right)$ denotes the maximum cardinality of any $\left(w, \varepsilon, Z, f_{0}, \ldots, f_{m-1}\right)$-separated sets of $Z$.

For any $n \geq 1$, let

$$
\begin{aligned}
& B\left(n, \varepsilon, Z, f_{0}, \ldots, f_{m-1}\right)=\frac{1}{m^{n}} \sum_{|w|=n} B\left(w, \varepsilon, Z, f_{0}, \ldots, f_{m-1}\right), \\
& N\left(n, \varepsilon, Z, f_{0}, \ldots, f_{m-1}\right)=\frac{1}{m^{n}} \sum_{|w|=n} N\left(w, \varepsilon, Z, f_{0}, \ldots, f_{m-1}\right) .
\end{aligned}
$$

Obviously,

$$
B\left(w, \varepsilon / 2, Z, f_{0}, \ldots, f_{m-1}\right) \geq N\left(w, \varepsilon, Z, f_{0}, \ldots, f_{m-1}\right) \geq B\left(w, \varepsilon, Z, f_{0}, \ldots, f_{m-1}\right)
$$

this implies that

$$
B\left(n, \varepsilon / 2, Z, f_{0}, \ldots, f_{m-1}\right) \geq N\left(n, \varepsilon, Z, f_{0}, \ldots, f_{m-1}\right) \geq B\left(n, \varepsilon, Z, f_{0}, \ldots, f_{m-1}\right) .
$$

Similar to [26], we can define

$$
\begin{aligned}
H\left(Z, f_{0}, \ldots, f_{m-1}\right) & =\lim _{\varepsilon \rightarrow 0} \limsup _{n \rightarrow \infty} \frac{1}{n} \log B\left(n, \varepsilon, Z, f_{0}, \ldots, f_{m-1}\right) \\
& =\lim _{\varepsilon \rightarrow 0} \limsup _{n \rightarrow \infty} \frac{1}{n} \log N\left(n, \varepsilon, Z, f_{0}, \ldots, f_{m-1}\right) .
\end{aligned}
$$

We write $H\left(Z, f_{0}, \ldots, f_{m-1}\right)$ as $H_{d}\left(Z, f_{0}, \ldots, f_{m-1}\right)$ to emphasize the metric $d$ if we need to. Then we have the following variational principle. 
Theorem 4.6. Let $X$ be an LCSMS. Suppose that $G$ is the free semigroup acting on $X$ generated by $\left\{f_{0}, f_{1}, \ldots, f_{m-1}\right\}$, where $f_{i}: X \rightarrow X(i=0,1, \ldots, m-1)$ are proper maps. Then for any $Z \subset X$, we have

$$
\overline{C h}_{Z}(G)=\min _{d} H_{d}\left(Z, f_{0}, \ldots, f_{m-1}\right),
$$

where the minimum is attained whenever $d$ is the metric given by the restriction to $X$ of some metric $\widetilde{d}$ on $\widetilde{X}$ the one-point compacification of $X$.

Lemma 4.7. 17] Let $(X, d)$ be a metric space, then every admissible cover of $X$ has a Lebesgue number.

Proof of Theorem 4.6. For every metric $d$ induced the topology of $X$. Let $\mathcal{U}$ be an admissible cover of $X$. Applying Lemma 4.7, $\mathcal{U}$ has a Lebesgue number denoted by $\delta$. Given $w \in F_{m}^{+}$with $|w|=n$, let $E \subset X$ be a $\left(w, \varepsilon, Z, f_{0}, \ldots, f_{m-1}\right)$-spanning set of $Z$ such that $2 \varepsilon<\delta$, then $\left\{B_{w}(x, \varepsilon): x \in E\right\}$ covers $Z$. For any $x \in E$, there exists a string $\mathbf{U} \in S_{n+1}(\mathcal{U})$ such that $B_{w}(x, \varepsilon) \subset X_{w}(\mathbf{U})$, it follows that

$$
\Lambda_{w}(Z, \mathcal{U}, n) \leq B\left(w, \varepsilon, Z, f_{0}, \ldots, f_{m-1}\right)
$$

Then

$$
\Lambda(Z, \mathcal{U}, n) \leq B\left(n, \varepsilon, Z, f_{0}, \ldots, f_{m-1}\right)
$$

By Proposition 3.4, we get that

$$
\overline{C h}_{Z}(\mathcal{U}, G) \leq \limsup _{n \rightarrow \infty} \frac{1}{n} \log B\left(n, \varepsilon, Z, f_{0}, \ldots, f_{m-1}\right) .
$$

Letting $\varepsilon \rightarrow 0$, we have

$$
\overline{C h}_{Z}(\mathcal{U}, G) \leq H_{d}\left(Z, f_{0}, \ldots, f_{m-1}\right) .
$$

Hence

$$
\overline{C h}_{Z}(G)=\sup _{\mathcal{U}} \overline{C h}_{Z}(\mathcal{U}, G) \leq H_{d}\left(Z, f_{0}, \ldots, f_{m-1}\right) .
$$

By the arbitrarily of the metric $d$, we have

$$
\overline{C h}_{Z}(G) \leq \inf _{d} H_{d}\left(Z, f_{0}, \ldots, f_{m-1}\right)
$$

On the other hand, if the metric $d^{\prime}$ is given by the restriction to $X$ of the $\widetilde{d}$ on $\widetilde{X}$. By Lemma $4.2, d^{\prime}$ is an admissible metric, and for any $\varepsilon>0$, there exists an admissible cover of $X$ such that the diameter of the cover is less than $\varepsilon$. Let $\mathcal{U}$ be an admissible cover with $|\mathcal{U}|<+\infty$. Given $w \in F_{m}^{+}$with $|w|=n$, assume $\mathcal{G}_{w} \subset S_{n+1}(\mathcal{U})$ covers $Z$. Fixing 
$x_{\mathbf{U}} \in X_{w}(\mathbf{U}), \forall \mathbf{U} \in \mathcal{G}$, and putting $E:=\left\{x_{\mathbf{U}}: \mathbf{U} \in \mathcal{G}_{w}\right\}$, we can easily see that $E$ is a $\left(w,|\mathcal{U}|, Z, f_{0}, \ldots, f_{m-1}\right)$-spanning set of $Z$. Moreover, we have that

$$
B\left(w,|\mathcal{U}|, Z, f_{0}, \ldots, f_{m-1}\right) \leq \Lambda_{w}(Z, \mathcal{U}, n),
$$

and

$$
B\left(n,|\mathcal{U}|, Z, f_{0}, \ldots, f_{m-1}\right) \leq \Lambda(Z, \mathcal{U}, n) .
$$

By Proposition 3.4, we get that

$$
\limsup _{n \rightarrow \infty} \frac{1}{n} \log B\left(n,|\mathcal{U}|, Z, f_{0}, \ldots, f_{m-1}\right) \leq \overline{C h}_{Z}(\mathcal{U}, G) .
$$

Applying Theorem 4.1 and letting $|\mathcal{U}| \rightarrow 0$, we obtain that

$$
H_{d^{\prime}}\left(Z, f_{0}, \ldots, f_{m-1}\right) \leq \overline{C h}_{Z}(G) .
$$

Combining (4.1), we have

$$
H_{d^{\prime}}\left(Z, f_{0}, \ldots, f_{m-1}\right)=\overline{C h}_{Z}(G)
$$

and the proof is complete.

Remark 4.8. Indeed, Ma et al. 17 proved that $\overline{C h}_{Z}(f)=\min _{d} H_{d}(Z, f)$, Patrão 20 proved that $\overline{C h}_{X}(f)=\min _{d} H_{d}(X, f)$. Then Theorem 4.6 extends the results of Ma et al. [17] and Patrão [20].

\section{Topological entropy and UCTE of skew-product transformations}

In this section, three skew-product transformations of proper maps is considered. We first study the relations of the entropies among these three skew-product transformations. For a particular subset, we then give the relationship between the UCTE of a free semigroup action generated by proper maps, and the UCTE of a skew-product transformation.

Let $X$ be an LCSMS, and its one-point compactification is denoted by $\widetilde{X}$. Let $f_{i}: X \rightarrow$ $X(i=0,1, \ldots, m-1)$ be proper maps and $\tilde{f}_{i}(i=0,1, \ldots, m-1)$ be its extension in the one-point compactification $\tilde{X}$ of $X$. The skew-product transformation [7] associated with $f_{i}(i=0,1, \ldots, m-1)$ is defined as

$$
F: \Sigma_{m} \times X \rightarrow \Sigma_{m} \times X, \quad F(\omega, x)=\left(\sigma_{m} \omega, f_{\omega_{0}}(x)\right) .
$$

Here $f_{\omega_{0}}$ stands for $f_{0}$ if $\omega_{0}=0$, and for $f_{1}$ if $\omega_{0}=1$, and so on. The skew-product transformation associated with $\widetilde{f}_{i}(i=0,1, \ldots, m-1)$ is defined as

$$
F^{*}: \Sigma_{m} \times \tilde{X} \rightarrow \Sigma_{m} \times \tilde{X}, \quad F^{*}(\omega, x)=\left(\sigma_{m} \omega, \tilde{f}_{\omega_{0}}(x)\right)= \begin{cases}\left(\sigma_{m} \omega, f_{\omega_{0}}(x)\right), & x \neq \infty \\ \left(\sigma_{m} \omega, \infty\right), & x=\infty\end{cases}
$$


We can easily get that the extension of $F$ is

$$
\widetilde{F}: \widetilde{\Sigma_{m} \times X} \rightarrow \widetilde{\Sigma_{m} \times X}, \quad \widetilde{F}(\omega, x)= \begin{cases}F(\omega, x), & (\omega, x) \in \Sigma_{m} \times X \\ \infty^{*}, & (\omega, x)=\infty^{*}\end{cases}
$$

where $\widetilde{\Sigma_{m} \times X}$ denotes the one-point compactification of $\Sigma_{m} \times X$ and $\infty^{*}$ is some point not in $\Sigma_{m} \times X$. It was shown in [24] that $F$ is a proper map, then it is easy to see that $F^{*}$ and $\widetilde{F}$ are also proper maps, thus we can study the relations of the entropies among these three skew-product transformations. First, we give the following two lemmas, the first of which generalizes Bowen inequality [5]. In the first lemma, by a topologically dynamical system $(X, T)$ (TDS) we mean a compact metric space $X$ with a continuous map $T$ from $X$ into itself.

Lemma 5.1. 111 Let $(X, T),(Y, S)$ be two TDSs, $\pi: X \rightarrow Y$ be a factor map between two TDSs (i.e., $\pi$ is a continuous surjective such that $\pi \circ T=S \circ \pi$ ). Then for any $E \subset X$,

$$
h_{\pi(E)}(S) \leq h_{E}(T) \leq h_{\pi(E)}(S)+\sup _{y \in Y} \overline{C h}_{\pi^{-1}(y)}(S)
$$

Lemma 5.2. For any set $Z \subset X$, we have

$$
h_{\Sigma_{m} \times Z}(\widetilde{F}) \leq h_{\Sigma_{m} \times Z}\left(F^{*}\right) \leq h_{\Sigma_{m} \times Z}(\widetilde{F})+\log m
$$

Proof. Defining $\pi: \Sigma_{m} \times \widetilde{X} \rightarrow \widetilde{\Sigma_{m} \times X}$ by

$$
\pi(\omega, x)= \begin{cases}(\omega, x), & x \neq \infty \\ \infty^{*}, & x=\infty\end{cases}
$$

Then it is easy to see that $\pi \circ F^{*}=\widetilde{F} \circ \pi$. By Lemma 5.1, we get that

$$
\begin{aligned}
h_{\Sigma_{m} \times Z}(\widetilde{F}) & \leq h_{\Sigma_{m} \times Z}\left(F^{*}\right) \leq h_{\Sigma_{m} \times Z}(\widetilde{F})+\underset{y \in \Sigma_{m \times X}}{\sup } \overline{C h_{\pi^{-1}(y)}}\left(F^{*}\right) \\
& =h_{\Sigma_{m} \times Z}(\widetilde{F})+\overline{C h}_{\pi^{-1}\left(\infty^{*}\right)}\left(F^{*}\right)=h_{\Sigma_{m} \times Z}(\widetilde{F})+\log m
\end{aligned}
$$

and the proof is complete.

Theorem 5.3. Suppose that $G$ is the free semigroup acting on $X$ generated by $\left\{f_{0}, f_{1}, \ldots\right.$, $\left.f_{m-1}\right\}$, then for any compact $G$-invariant set $Z \subset X$, we have

$$
\overline{C h}_{Z}(G) \leq \overline{C h}_{\Sigma_{m} \times Z}(F) \leq \overline{C h}_{Z}(G)+\log m .
$$


Proof. $Z$ is a compact $G$-invariant subset of $X$, this implies that $\Sigma_{m} \times Z$ is a compact $F$-invariant subset of $\Sigma_{m} \times X$ and a compact $F^{*}$-invariant subset of $\Sigma_{m} \times \tilde{X}$. According to [21, Theorem 11.5] and [17, Theorem 4.1], we have

$$
h_{\Sigma_{m} \times Z}\left(F^{*}\right)=\overline{C h}_{\Sigma_{m} \times Z}\left(F^{*}\right) \quad \text { and } \quad h_{\Sigma_{m} \times Z}(F)=\overline{C h}_{\Sigma_{m} \times Z}(F) .
$$

Since $\widetilde{X}$ is compact, by $[13$, Theorem 5.1] and Theorem 4.4 , we have

$$
\overline{C h}_{\Sigma_{m} \times Z}\left(F^{*}\right)=\overline{C h}_{Z}(\widetilde{G})+\log m=\overline{C h}_{Z}(G)+\log m .
$$

By Lemma 5.2, we get that

$$
\overline{C h}_{\Sigma_{m} \times Z}(\widetilde{F})=h_{\Sigma_{m} \times Z}(\widetilde{F}) \leq h_{\Sigma_{m} \times Z}\left(F^{*}\right)=\overline{C h}_{\Sigma_{m} \times Z}\left(F^{*}\right)=\overline{C h}_{Z}(G)+\log m
$$

and

$$
\begin{aligned}
\overline{C h}_{Z}(G)+\log m & =\overline{C h}_{\Sigma_{m} \times Z}\left(F^{*}\right)=h_{\Sigma_{m} \times Z}\left(F^{*}\right) \\
& \leq h_{\Sigma_{m} \times Z}(\widetilde{F})+\log m=\overline{C h}_{\Sigma_{m} \times Z}(\widetilde{F})+\log m .
\end{aligned}
$$

Hence

$$
\overline{C h}_{Z}(G) \leq \overline{C h}_{\Sigma_{m} \times Z}(\widetilde{F}) \leq \overline{C h}_{Z}(G)+\log m .
$$

Moreover, by [17, Theorem 4.3], we have

$$
\overline{C h}_{\Sigma_{m} \times Z}(F)=\overline{C h}_{\Sigma_{m} \times Z}(\widetilde{F}) .
$$

Thus we obtain the result immediately.

Remark 5.4. Let $X$ be a compact metric space and $Z=X$, Tian et al. [24] proved that $h_{X}(G) \leq h_{\Sigma_{m} \times X}(F) \leq h_{X}(G)+\log m$, this is a special case of Theorem 5.3 .

\section{Applications}

In this section, we study some applications of the topological entropies proposed in this paper when $X$ is an LCSMS. We first prove that the multifractal spectrum of an LCSMS is equal to that of its one-point compactification. Inspired by Zhu and Ma 29], then we focus on the UCTE of the irregular set for an LCSMS.

\subsection{Multifractal spectrum}

The following general concept of multifractal spectra was introduced in [2]. Let $X$ be a set and let $g: X \rightarrow[-\infty,+\infty]$ be a function. The level sets of $g$

$$
K_{\alpha}^{g}=\{x \in X: g(x)=\alpha\}, \quad-\infty \leq \alpha \leq+\infty
$$


are disjoint and produce a multifractal decomposition of $X$, that is,

$$
X=\bigcup_{-\infty \leq \alpha \leq+\infty} K_{\alpha}^{g}
$$

Let $F$ be a set function (i.e., a real function that is defined on subsets of $X$ ), satisfying that $F\left(Z_{1}\right) \leq F\left(Z_{2}\right)$ if $Z_{1} \subset Z_{2}$. Define the function $\mathcal{F}:[-\infty,+\infty] \rightarrow \mathbb{R}$ by $\mathcal{F}(\alpha)=$ $F\left(K_{\alpha}^{g}\right)$, we call $\mathcal{F}$ the multifractal spectrum specified by the pair of functions $(g, F)$, or the $(g, F)$-multifractal spectrum.

It happens that the function $g$ is defined only on a subset $Y \subset X$. In this case the decomposition (6.1) should be replaced by

$$
X=\bigcup_{-\infty \leq \alpha \leq+\infty} K_{\alpha}^{g} \cup(X \backslash Y) .
$$

We still call this decomposition of $X$ a multifractal decomposition.

The function $\mathcal{F}$ captures an important information about the structure of the set $X$ generated by the function $g$. Then we have the following theorem.

Theorem 6.1. Let $X$ be an LCSMS. Let $f_{i}: X \rightarrow X(i=0,1, \ldots, m-1)$ be proper maps and $\widetilde{f}_{i}(i=0,1, \ldots, m-1)$ be its extension of $f_{i}$ to $\widetilde{X}$. Suppose that $G$ and $\widetilde{G}$ are the free semigroups acting on $X$ and $\widetilde{X}$, generated by $\left\{f_{0}, f_{1}, \ldots, f_{m-1}\right\}$ and $\left\{\widetilde{f}_{0}, \widetilde{f}_{1}, \ldots, \widetilde{f}_{m-1}\right\}$ respectively. Let $g: X \rightarrow[-\infty,+\infty]$ be a function and $\widetilde{g}: \widetilde{X} \rightarrow[-\infty,+\infty]$ be any extension of $g$. If the set functions $F$ and $\widetilde{F}$ are defined as $F(Z)=h_{Z}(G)(\forall Z \subset X)$ and $\widetilde{F}(\widetilde{Z})=$ $h_{\widetilde{Z}}(\widetilde{G})(\forall \widetilde{Z} \subset \widetilde{X})$ respectively, then $X$ and $\widetilde{X}$ have the same multifractal spectrum specified by $(g, F)$ and $(\widetilde{g}, \widetilde{F})$ respectively.

Proof. Let $\alpha \in[-\infty,+\infty]$, then

$$
K_{\alpha}^{\widetilde{g}}=\{x \in \widetilde{X}: \widetilde{g}(x)=\alpha\}=\{x \in X: g(x)=\alpha\}=K_{\alpha}^{g},
$$

or

$$
K_{\alpha}^{\widetilde{g}}=\{x \in \widetilde{X}: \widetilde{g}(x)=\alpha\}=\{x \in X: g(x)=\alpha\} \cup\{\infty\}=K_{\alpha}^{g} \cup\{\infty\},
$$

where $\infty$ is the point in the definition of $\widetilde{X}$. Note that $h_{\{\infty\}}(\widetilde{G})=0$, by Proposition $3.2(3)$, we have

$$
h_{K_{\alpha}^{\widetilde{g}}}(\widetilde{G})=h_{K_{\alpha}^{g}}(\widetilde{G})
$$

By Theorem 4.4, we get that

$$
h_{K_{\alpha}^{g}}(G)=h_{K_{\alpha}^{g}}(\widetilde{G})=h_{K_{\alpha}^{\widetilde{g}}}(\widetilde{G})
$$

and the theorem is proved. 


\subsection{Irregularity}

Let $X$ be a metric space with metric $d$ and $f_{i}: X \rightarrow X(i=0,1,2, \ldots, m-1)$ be proper maps. $G$ is the free semigroup acting on $X$ generated by $\left\{f_{0}, f_{1}, \ldots, f_{m-1}\right\}$. Let $\varphi: X \rightarrow \mathbb{R}$ be a continuous function, a point $x \in X$ is called to be $\varphi$-irregular point of a free semigroup action for $\varphi 29$ if there exists $\omega=\left(i_{1} i_{2} \cdots\right) \in \Sigma_{m}^{+}$, the $\operatorname{limit}_{\lim } \rightarrow \infty \frac{1}{n} \sum_{j=1}^{n} \varphi\left(f_{i_{j}, \ldots, i_{1}}(x)\right)$ does not exist. Let $I_{\varphi}(G)$ denote the set of all $\varphi$-irregular points of a free semigroup action. The union $I(G)=\bigcup_{\varphi \in C(X, \mathbb{R})} I_{\varphi}(G)$ is called the irregular set of a free semigroup action.

We say that $G$ has the specification property $[22$ if for any $\delta>0$, there exists $p(\delta)>0$, such that for any $k>0$, any points $x_{1}, \ldots, x_{k} \in X$, any sequences of positive integers $n_{1}, \ldots, n_{k}$ and any $p_{1}, \ldots, p_{k} \geq p(\delta)$, and any $w_{\left(p_{j}\right)} \in F_{m}^{+}$with $\left|w_{\left(p_{j}\right)}\right|=p_{j}, w_{\left(n_{j}\right)} \in F_{m}^{+}$ with $\left|w_{\left(n_{j}\right)}\right|=n_{j}, 1 \leq j \leq k$, there exists $x \in X$ such that

$$
d\left(f_{w}(x), f_{w}\left(x_{1}\right)\right)<\delta, \quad \forall w \leq \overline{w_{\left(n_{1}\right)}},
$$

and

$$
d\left(f_{w} f_{\overline{w_{\left(p_{j-1}\right)}}} f_{\overline{w_{\left(n_{j-1}\right)}}} \cdots f_{\overline{w_{\left(p_{1}\right)}}} f_{\overline{w_{\left(n_{1}\right)}}}(x), f_{w}\left(x_{j}\right)\right)<\delta, \quad \forall w \leq \overline{w_{\left(n_{j}\right)}}, 2 \leq j \leq k .
$$

With the above basic concepts, we give the following theorem.

Theorem 6.2. Let $X$ be an LCSMS and $f_{i}: X \rightarrow X(i=0,1,2, \ldots, m-1)$ be proper maps. Let $d$ be the metric given by the restriction to $X$ of some metric $\widetilde{d}$ on $\widetilde{X}$. Suppose that $G$ is the free semigroup acting on $X$ generated by $\left\{f_{0}, f_{1}, \ldots, f_{m-1}\right\}$ and $G$ has specification property. Let $\varphi \in C(X, \mathbb{R})$ be bounded and can be continuously extended to $\widetilde{X}$. Let $I_{\varphi}(G)$ denote the set of all $\varphi$-irregular points of $G$, then either $I_{\varphi}(G)=\emptyset$ or

$$
\overline{C h}_{I_{\varphi}(G)}(G)=\overline{C h}_{X}(G)=h_{X}(G) \text {. }
$$

Before proving this theorem, we give the following two lemmas.

Lemma 6.3. 29] Let $X$ be a compact metric space. $G$ is the free semigroup acting on $X$ generated by $\left\{f_{0}, f_{1}, \ldots, f_{m-1}\right\}$, where $f_{i}: X \rightarrow X(i=0,1, \ldots, m-1)$ are continuous, and $G$ has the specification property. Let $\varphi: X \rightarrow \mathbb{R}$ be a continuous function. Then either $I_{\varphi}(G)=\emptyset$ or

$$
\overline{C h}_{I_{\varphi}(G)}(G)=\overline{C h}_{X}(G)=h_{X}(G) .
$$

Lemma 6.4. Let $X$ be an LCSMS with metric $d$ which is the restriction of some metric $\tilde{d}$ on $\tilde{X}$. Let $f_{i}: X \rightarrow X(i=0,1, \ldots, m-1)$ be proper maps and $\widetilde{f}_{i}(i=0,1, \ldots, m-$ 1 ) be its extension of $f_{i}$ to $\tilde{X} . G$ and $\widetilde{G}$ are the free semigroups acting on $X$ and $\tilde{X}$ respectively, generated by $\left\{f_{0}, f_{1}, \ldots, f_{m-1}\right\}$ and $\left\{\widetilde{f}_{0}, \widetilde{f}_{1}, \ldots, \widetilde{f}_{m-1}\right\}$ respectively. If $G$ has the specification property, then $\widetilde{G}$ also has the specification property. 
Proof. For any $\delta>0$, there exists $p(\delta)>0$, such that for any $k>0$, any points $x_{1}, \ldots, x_{k} \in$ $X$, any sequences of positive integers $n_{1}, \ldots, n_{k}$ and any $p_{1}, \ldots, p_{k} \geq p(\delta)$, and any $w_{\left(p_{j}\right)} \in$ $F_{m}^{+}$with $\left|w_{\left(p_{j}\right)}\right|=p_{j}, w_{\left(n_{j}\right)} \in F_{m}^{+}$with $\left|w_{\left(n_{j}\right)}\right|=n_{j}, 1 \leq j \leq k$, there exists $x \in X$ such that

$$
d\left(f_{w}(x), f_{w}\left(x_{1}\right)\right)<\delta, \quad \forall w \leq \overline{w_{\left(n_{1}\right)}},
$$

and

$$
d\left(f_{w} f_{\overline{w_{\left(p_{j-1}\right)}}} f_{\overline{w_{\left(n_{j-1}\right)}}} \cdots f_{\overline{w_{\left(p_{1}\right)}}} f_{\overline{w_{\left(n_{1}\right)}}}(x), f_{w}\left(x_{j}\right)\right)<\delta, \quad \forall w \leq \overline{w_{\left(n_{j}\right)}}, 2 \leq j \leq k
$$

If $\widetilde{x}_{1}, \ldots, \widetilde{x}_{k} \in \widetilde{X}$ and $\widetilde{x}_{i}=\infty, 1 \leq i \leq k$. It is sufficient to consider the case of $i=1$. Putting $\widetilde{p}(\delta)=p(\delta / 2)$, for any sequences of positive integers $n_{1}, \ldots, n_{k}$ and any $p_{1}, \ldots, p_{k} \geq \widetilde{p}(\delta)$, and any $w_{\left(p_{j}\right)} \in F_{m}^{+}$with $\left|w_{\left(p_{j}\right)}\right|=p_{j}, w_{\left(n_{j}\right)} \in F_{m}^{+}$with $\left|w_{\left(n_{j}\right)}\right|=n_{j}$, $1 \leq j \leq k$, by the density of $X$ in $\widetilde{X}$ and the uniform continuity of $\widetilde{f}_{0}, \widetilde{f}_{1}, \ldots, \widetilde{f}_{m-1}$, there exists $x_{1} \in X$ such that

$$
\widetilde{d}\left(f_{w}\left(\widetilde{x}_{1}\right), f_{w}\left(x_{1}\right)\right)<\frac{\delta}{2}, \quad \forall w \leq \overline{w_{\left(n_{1}\right)}} .
$$

For $x_{1}, \widetilde{x}_{2}, \ldots, \widetilde{x}_{k} \in X$, and above $n_{j}, p_{j}, w_{\left(n_{j}\right)}, w_{\left(p_{j}\right)}, 1 \leq j \leq k$, there exists a point $z \in X$ such that

$$
\widetilde{d}\left(f_{w}(z), f_{w}\left(x_{1}\right)\right)<\frac{\delta}{2}, \quad \forall w \leq \overline{w_{\left(n_{1}\right)}}
$$

and

$$
\widetilde{d}\left(f_{w} f_{\overline{w_{\left(p_{j-1}\right)}}} f_{\overline{w_{\left(n_{j-1}\right)}}} \cdots f_{\overline{w_{\left(p_{1}\right)}}} f_{\overline{w_{\left(n_{1}\right)}}}(z), f_{w}\left(\widetilde{x}_{j}\right)\right)<\frac{\delta}{2}, \quad \forall w \leq \overline{w_{\left(n_{j}\right)}}, 2 \leq j \leq k .
$$

Moreover,

$$
\widetilde{d}\left(f_{w}(z), f_{w}\left(\widetilde{x}_{1}\right)\right)<\frac{\delta}{2}+\frac{\delta}{2}=\delta, \quad \forall w \leq \overline{w_{\left(n_{1}\right)}}
$$

and

$$
\widetilde{d}\left(f_{w} f_{\overline{w_{\left(p_{j-1}\right)}}} f_{\overline{w_{\left(n_{j-1}\right)}}} \cdots f_{\overline{w_{\left(p_{1}\right)}}} f_{\overline{w_{\left(n_{1}\right)}}}(z), f_{w}\left(\widetilde{x}_{j}\right)\right)<\frac{\delta}{2}<\delta, \quad \forall w \leq \overline{w_{\left(n_{j}\right)}}, 2 \leq j \leq k .
$$

Therefore, $\widetilde{G}$ has the specification property.

Proof of Theorem 6.2. We just consider the case of $I_{\varphi}(G) \neq \emptyset$. Let $\widetilde{f}_{i}$ be the extension of $f_{i}$ to $\widetilde{X}(i=0,1, \ldots, m-1)$, then it is easy to see that $\widetilde{f}_{i}(i=0,1, \ldots, m-1)$ are continuous. Suppose that $\widetilde{G}$ is the free semigroup acting on $\widetilde{X}$ generated by $\left\{\widetilde{f}_{0}, \widetilde{f}_{1}, \ldots, \widetilde{f}_{m-1}\right\}$. By Lemma 6.4. $\widetilde{G}$ has the specification property. Let $\widetilde{\varphi} \in C(\widetilde{X}, \mathbb{R})$ be an extension of $\varphi$, then $I_{\widetilde{\varphi}}(\widetilde{G})=I_{\varphi}(G)$ or $I_{\widetilde{\varphi}}(\widetilde{G})=I_{\varphi}(G) \cup\{\infty\}$. It is easy to see that

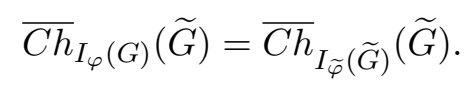


By Theorem 4.4 and Lemma 6.3, we get that

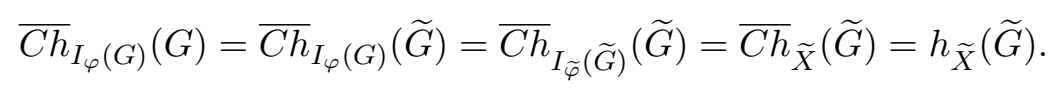

Moreover, combining the fact that $\tilde{X}=X \cup\{\infty\}$ and Theorem 4.4 , we can easily verify that

$$
\overline{C h}_{\widetilde{X}}(\widetilde{G})=\overline{C h}_{X}(G) \quad \text { and } \quad h_{\widetilde{X}}(\widetilde{G})=h_{X}(G) .
$$

Thus we get the result immediately.

Remark 6.5. If $X$ is a compact metric space, under the same conditions as that of Theorem 6.2. Zhu and Ma 29] proved Lemma 6.3. Then Theorem 6.2 is an extension of Lemma 6.3

By 29, Corollary 4.2] and Theorem 6.2, we have the following corollary.

Corollary 6.6. Let $X$ be an LCSMS and $f_{i}: X \rightarrow X(i=0,1,2, \ldots, m-1)$ be proper maps. Let $d$ be the metric given by the restriction to $X$ of some metric $\widetilde{d}$ on $\widetilde{X}$. Suppose that $G$ is the free semigroup acting on $X$ generated by $\left\{f_{0}, f_{1}, \ldots, f_{m-1}\right\}$ and $G$ has specification property. Let $I(G)=\bigcup_{\varphi \in C(X, \mathbb{R})} I_{\varphi}(G)$ be the irregular set of a free semigroup action, then either $I(G)=\emptyset$ or

$$
\overline{C h}_{I(G)}(G)=\overline{C h}_{X}(G)=h_{X}(G) .
$$

\section{Acknowledgments}

The authors really appreciate the editor and referees' valuable remarks and suggestions that helped a lot. The work was supported by National Natural Science Foundation of China (grant no. 11771149).

\section{References}

[1] R. L. Adler, A. G. Konheim and M. H. McAndrew, Topological entropy, Trans. Amer. Math. Soc. 114 (1965), 309-319.

[2] L. Barreira, Y. Pesin and J. Schmeling, On a general concept of multifractality: Multifractal spectra for dimensions, entropies, and Lyapunov exponentS, multifractal rigidity, Chaos 7 (1997), no. 1, 27-38.

[3] A. Biś, Entropies of a semigroup of maps, Discrete Contin. Dyn. Systs. 11 (2004), no. 2-3, 639-648. 
[4] A. Biś, D. Dikranjan, A. Giordano Bruno and L. Stoyanov, Topological entropy, upper Carathéodory capacity and fractal dimensions of semigroup actions, Colloq. Math. 163 (2021), no. 1, 131-151.

[5] R. Bowen, Entropy for group endomorphisms and homogeneous spaces, Trans. Amer. Math. Soc. 153 (1971), 401-414.

[6] - Topological entropy for noncompact sets, Trans. Amer. Math. Soc. 184 (1973), 125-136.

[7] A. Bufetov, Topological entropy of free semigroup actions and skew-product transformations, J. Dynam. Control Systems 5 (1999), no. 1, 137-143.

[8] C. Carathéodory, Über das Lineare Mass, Göttingen Nachr, (1914), 406-426.

[9] M. Carvalho, F. B. Rodrigues and P. Varandas, Semigroup actions of expanding maps, J. Stat. Phys. 166 (2017), no. 1, 114-136.

[10] C. Ercai, T. Küpper and S. Lin, Topological entropy for divergence points, Ergodic Theory Dynam. Systems 25 (2005), no. 4, 1173-1208.

[11] C. Fang, W. Huang, Y. Yi and P. Zhang, Dimensions of stable sets and scrambled sets in positive finite entropy systems, Ergodic Theory Dynam. Systems 32 (2012), no. 2, 599-628.

[12] É. Ghys, R. Langevin and P. Walczak, Entropie géométrique des feuilletages, Acta Math. 160 (1988), no. 1-2, 105-142.

[13] Y. Ju, D. Ma and Y. Wang, Topological entropy of free semigroup actions for noncompact sets, Discrete Contin. Dyn. Syst. 39 (2019), no. 2, 995-1017.

[14] P. E. Kloeden and M. Rasmussen, Nonautonomous Dynamical Systems, Mathematical Surveys and Monographs 176, American Mathematical Society, Providence, RI, 2011.

[15] Z. Li, Z. Ding and Y. Yang, Sub-additive topological pressure of proper maps, Dyn. Syst. 33 (2018), no. 3, 464-479.

[16] X. Lin, D. Ma and Y. Wang, On the measure-theoretic entropy and topological pressure of free semigroup actions, Ergodic Theory Dynam Systems 38 (2018), no. 2, 686-716.

[17] D. Ma and B. Cai, Topological entropy of proper map, Taiwanese J. Math. 18 (2014), no. $4,1219-1241$.

[18] D. Ma and N. Fan, Topological pressure of proper map, Chaos Solitons Fractals 105 (2017), 137-144. 
[19] D. Ma and M. Wu, Topological pressure and topological entropy of a semigroup of maps, Discrete Contin. Dyn. Syst. 31 (2011), no. 2, 545-557.

[20] M. Patrão, Entropy and its variational principle for non-compact metric spaces, Ergodic Theory Dynam. Systems 30 (2010), no. 5, 1529-1542.

[21] Y. B. Pesin, Dimension Theory in Dynamical Systems, Chicago Lectures in Mathematics, University of Chicago Press, Chicago, IL, 1997.

[22] F. B. Rodrigues and P. Varandas, Specification and thermodynamical properties of semigroup actions, J. Math. Phys. 57 (2016), no. 5, 052704, 27 pp.

[23] X. Tian, Different asymptotic behavior versus same dynamical complexity: Recurrence \& (ir)regularity, Adv. Math. 288 (2016), 464-526.

[24] Y. Tian, D. Ma and X. Lin, On the topological entropy of the free semigroup action generated by proper maps, J. Difference Equ. Appl. 24 (2018), no. 7, 1145-1163.

[25] Y. Wang and D. Ma, On the topological entropy of a semigroup of continuous maps, J. Math. Anal. Appl. 427 (2015), no. 2, 1084-1100.

[26] Y. Wang, D. Ma and X. Lin, On the topological entropy of free semigroup actions, J. Math. Anal. Appl. 435 (2016), no. 2, 1573-1590.

[27] Q. Xiao and D. Ma, Topological pressure of free semigroup actions for non-compact sets and Bowen's equation I, to appear in J. Dynam. Differential Equations, 2021. https://doi.org/10.1007/s10884-021-09983-3

[28] _ Topological pressure of free semigroup actions for non-compact sets and Bowen's equation II, to appear in J. Dynam. Differential Equations, 2021. https://doi.org/10.1007/s10884-021-10055-9

[29] L. Zhu and D. Ma, The upper capacity topological entropy of free semigroup actions for certain non-compact sets, J. Stat. Phys. 182 (2021), no. 1, Paper No. 19, 22 pp.

Xiaoyi Xie and Dongkui Ma

School of Mathematics, South China University of Technology, Guangzhou 510641, China E-mail addresses: $1549718341 @ q q . c o m$, dkma@scut.edu.cn 